\title{
Themenheft „Asthma“ der Sektion Pneumologie in der DGAI
}

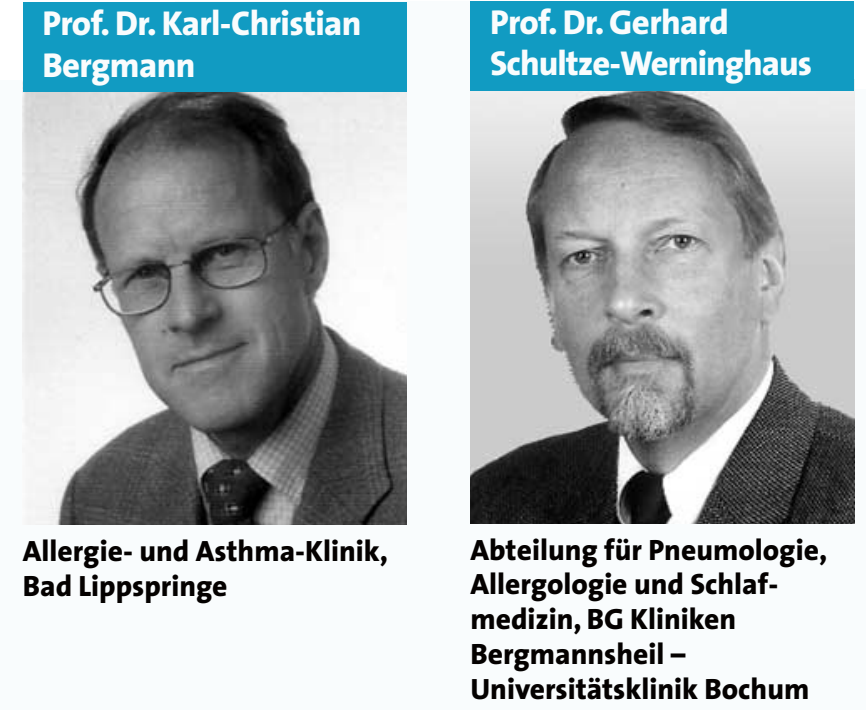

husten bis zur schweren anhaltenden Atemnot mit tief empfundenen Ängsten und schwer reduzierter Lebensqualität reicht.

\section{Ein aktueller Blick auf das Asthma bronchiale}

Asthma wurde über die Jahrhunderte immer wieder anders gesehen, verstanden und therapiert. Die Sektion Pneumologie in der DGAI will mit dem Inhalt dieses und des nächsten Themenhefts des Allergo Journals auf einige der wesentlichen neuen Gesichtspunkte im Verständnis des Asthma, seiner Diagnostik und Therapie hinweisen.

Selbstverständlich sind keineswegs alle Asthma-Patienten Allergiker. Aber das Risiko, an Asthma zu erkranken, nimmt mit der Höhe des Gesamt-IgE zu. Besonders die frühkindliche inhalative Belastung mit Allergenen von Hausstaubmilben, Tierhaaren und Pollen ist mit dem Auftreten von Sensibilisierungen gegen Inhalationsallergene eng verknüpft - eine Allergenbelastung im frühen Kindesalter erhöht das Risiko, an Asthma zu erkran- ken. Von etwa 4 Millionen behandlungsbedürftigen Asthmaerkrankungen in Deutschland ist im Kindes- und Jugendalter der größere und im Erwachsenenalter ein geringerer Teil auf eine klinisch aktuelle Sensibilisierung gegen Inhalationsallergene zurückzuführen.

Durch die typischen Symptome von anfallsweise auftretender Atemnot, häufig nachts und am frühen Morgen, den trockenen Husten, das charakteristische Giemen und ein glasig-zähes Sputum ist Asthma häufig bereits ohne weitere Diagnostik verdachtsweise zu erkennen. D. Menz stellt auf übersichtliche Weise die weit darüber hinaus gehenden Möglichkeiten in der Diagnostik des Asthma zusammen und verweist neben den herkömmliim frühen Kindesalter erhöht das Risiko an Asthma zu erkranken ... “"

chen Lungenfunktionsprüfungen und den wichtigen Provokationstests auch auf die nicht invasive Beurteilung der Atemwegsentzündung durch die Messung von Gasen und Kondensaten der Ausatmungsluft sowie durch Sputum- untersuchungen. Besonders die Kondensatuntersuchungen haben in den letzten fünf Jahren enorme Fortschritte gemacht und können möglicherweise bald in die klinische Routine aufgenommen werden.

\section{Genetischen Hintergrund beleuchten}

Dass Asthma offensichtlich in bestimmten Familien gehäuft auftritt, ist eine klinische Erfahrung vieler Ärztegenerationen. Beckett und Holgate geben in ihrer Arbeit einen ausgezeichneten Überblick über die gegenwärtigen Kenntnisse zur Bedeutung der genetischen Faktoren beim Asthma. Die Autoren haben es verstanden, die scheinbar verwirrende Fülle an Daten so zusammenzufassen, dass die Komplexität des Problems erkennbar und die Richtung der genetischen Forschung verständlich werden.

\section{Aktuelle Therapieansätze}

Die Grundlagenforschung führt beim Asthma zu einer immer tiefer gehenden Einsicht in die biochemischen und molekularbiologischen Abläufe in der $\mathrm{Pa}$ thogenese. Darauf aufbauend werden durch forschende Pharmaunternehmen neue Therapieansätze entwickelt. Dabei handelt es sich sowohl um Weiterentwicklungen bekannter Substanzen bzw. die feste Kombination von z. B. Steroiden mit langwirksamen Betamimetika als auch um die Entwicklung von Inhibitoren von Chemokinen oder Zytokinen bzw. von Anti-IgE-Antikörpern. Gillissen gibt eine Übersicht über die bereits erreichten und die möglicherweise zu erwartenden neuen Therapieansätze. Dem Leser dieser Arbeit wird 


\section{ZUR SACHE Themenheft Asthma}

Editor's Note

deutlich, dass wir einer immer gezielteren Asthmatherapie entgegengehen.

\section{Notfällen vorbeugen und Notfälle managen}

Durch die in den letzten Jahrzehnten verstärkten Bemühungen um verbesserte Asthmapräparate sowie deren sinnvolle und in nationalen Empfehlungen verbreitete Anwendung im Kontext mit verstärkter Einbeziehung des Patienten (Patientenschulungsprogramme) sowie stärkere Bemühungen bereits um die Vermeidung von Asthmaauslösern durch eine gute Allergiediagnostik hat sich die Zahl schwerer Asthmaanfälle in Deutschland vermindert, darstellbar an der geringeren Zahl von Patienten, die als Notfall auf Intensivstationen aufgenommen werden müssen.

Trotz aller Bemühungen aber erleiden noch zu viele Asthmatiker einen schweren Asthmaanfall, und es ist der Verdienst von Rasche und Mitarbeitern, Ursachen und Therapie in sehr übersichtlicher Form dargestellt zu haben.

\section{Stationäre Rehabilitation immer noch überlegen}

Eine gezielte Primärprävention (für Allergologen bedeutet dies die Vermeidung von Sensibilisierungen) ist beim Asthma noch nicht möglich, die Sekundärprävention durch Allergenkarenz, spezifische Immuntherapie, Patientenschulung und eine rationale medikamentöse Therapie sind wichtig. Alle diese Faktoren sollen in ein Gesamtmanagement des Asthma einbezogen werden. Leider aber müssen noch immer in vielen Fällen Maßnahmen der Tertiärprävention ergriffen werden, d. h. Rehabilitation von Patienten mit Asthma. Die pneumologische Rehabilitation des Asthma ist ein Prozess, der systematisch wissenschaftlich untermauerte diagnostische und therapeutische Verfahren nutzt, um die optimale tägliche Funktion und krankheitsbezogene Lebensqualität für den einzelnen Patienten zu erreichen. Zielstellung, Möglichkeiten und Verfahrensweise der stationären Rehabilitation von Asthmatikern werden durch Schultz und Mitarbeiter übersichtlich dargestellt. Die wohnortnahe ambulante Rehabilitation hat bis heute noch nicht die Effekte erzielen können, die bei der stationären Rehabilitation von Asthmatikern erreicht werden.

\section{Ein Blick durch die Jahrhunderte}

Vor mehr als 20 Jahren (Januar 1980) wurde durch Dietrich Nolte in Bad Reichenhall das nun bereits in fünfter Auflage vorliegende Buch mit dem schlichten Titel "Asthma“ herausgegeben [2],

Ihr

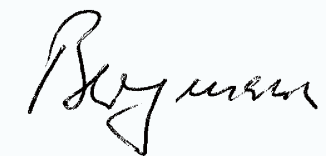

Prof. Dr. med. K.-Ch. Bergmann
Themenschwerpunkt Asthma -

Fortsetzung in Ausgabe 4/2002

In der nächsten Ausgabe des Allergo Journals finden Sie die Beiträge

Beckett PA, Holgate ST:

Genetics of Asthma

Rasche K, Schultze-Werninghaus G: Der schwere Asthmaanfall - Ursachen und Therapie

Schultz K:

Rehabilitation bei Asthma bronchiale

- Stellenwert und Verfahren

Die Ausgabe 4/2002 erscheint am 7. Juni 2002.

und es gibt keinen besseren Sachkenner - auch für die Geschichte der Asthmatherapie - als Herrn Nolte. Die Herausgeber der Themenhefte sind dankbar, dass er es übernommen hat, auf eine sehr interessante Weise die Entwicklung der Asthmatherapie von den ersten Anfängen an bis in unsere Zeit zu veranschaulichen.

Ihr

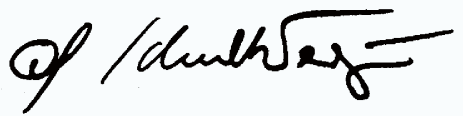

Prof. Dr. med. G. Schultze-Werninghaus
1. Wettengel $R$ et al. Empfehlungen zur Asthmatherapie bei Kindern und Erwachsenen. Pneumologie 1998; 52: 591-601

2. Nolte D. Asthma. Urban und Schwarzenberg, München, 1. Auflage 1980 